\title{
Preacondicionamiento isquémico remoto sobre viabilidad del injerto hepático
}

\author{
Remote ischemic preconditioning in liver graft viability
}

Paula Cordero-Pérez', Marco Hernández-Guedea², Julio C. Jiménez-Pérez', Linda Muñoz-Espinosa', Edelmiro Pérez-Rodríguez ${ }^{2}$ y Homero A. Zapata-Chavira ${ }^{2 *}$

${ }^{1}$ Departamento de Medicina Interna; ${ }^{2}$ Servicio de Trasplantes. Universidad Autónoma de Nuevo León, Hospital Universitario Dr. José E. González, Monterrey, Nuevo León, México

\section{Resumen}

Antecedentes: El preacondicionamiento isquémico remoto (PIR) en trasplante hepático ha sido sugerido en el ámbito experimental como estrategia para disminuir la lesión por isquemia- reperfusión. Objetivo: Evaluar el efecto del PIR sobre el injerto hepático en donante cadáver y el impacto de diversos mediadores inflamatorios en este proceso. Método: Se incluyeron 10 receptores de trasplante hepático, 5 controles y 5 con PIR, el cual fue realizado en los donantes cadavéricos mediante la aplicación de un torniquete neumático en ambos muslos por 10 minutos seguido de 10 minutos de reperfusión. Se determinaron interleucina (IL)-1, IL-6, factor de necrosis tumoral alfa (FNT- $\alpha$ ), factor de crecimiento endotelial vascular (FCEV) y molécula de adhesión intracelular (ICAM)-1, parámetros hematológicos y bioquímicos en diversas fases del trasplante hepático. Resultados: Se observó un aumento significativo de la aspartato aminotransferasa (AST), la alanino aminotransferasa $(A L T)$ y la fosfatasa alcalina en las fases tempranas tras el trasplante hepático, y a las 72 horas los sujetos con PIR mostraron mejor respuesta, con recuperación de plaquetas, que persistió hasta los 3 meses en este grupo. La IL-6 participa en las fases tempranas de la lesión por isquemia- reperfusión, contrario al FNT- $\alpha$, que se incrementa hasta el día 7, mientras que la ICAM-1 aumentó en todas las fases. Conclusiones: En este estudio piloto, el PIR disminuyó el daño por lesión por isquemia- reperfusión, aunque el mayor efecto se observó después de 72 horas.

PALABRAS CLAVE: Isquemia/reperfusión. Trasplante hepático. Preacondicionamiento isquémico. Preacondicionamiento isquémico remoto.

\begin{abstract}
Background: Remote ischemic preconditioning (RIP) in liver transplantation has been suggested experimentally as a strategy to reduce ischemia-reperfusion injury. Objective: Evaluate the effect of RIP on liver graft in cadaveric donors and the impact of various inflammatory mediators in this process. Method: Ten liver transplantation recipients, 5 controls and 5 PIR, were made in the cadaver donors by applying a pneumatic tourniquet in the upper third of both thighs for a period of 10 minutes followed by 10 minutes reperfusion. The determination of interleukine (IL)-1, IL-6, tumor necrosis factor alpha (TNF- - ), vascular endothelial growth factor (VEGF), intracellular adhesion molecule (ICAM)-1 was performed as well as hematological and biochemical parameters at various stages of liver transplantation. Results: Significant increase of aspartate aminotransferase (AST), alanine aminotransferase $(A L T)$ and alkaline phosphatase in the early stages of post-liver transplantation were observed, after 72 hours subjects who received liver transplantation subjected to RIP they showed a better response, which was also evident in platelet recovery, which persisted until phase 3 months in this group. IL-6 appears to participate in the
\end{abstract}

Correspondencia:

Homero A. Zapata-Chavira

Gonzalitos 235, Col. Mitras Centro, C.P. 64460,

Fecha de recepción: 12-04-2018

Cir Cir. 2018;86:539-547

Monterrey, N.L., México

Fecha de aceptación: 15-05-2018

E-mail: homero_zapata@yahoo.com

DOI: 10.24875/CIRU.18000336

Contents available at PubMed

www.cirugiaycirujanos.com 
early stages of the ischemia-reperfusion injury, contrary to TNF- $\alpha$ that increases until day 7 while ICAM-1 was increased in all phases. Conclusions: In this pilot study the PIR decreased the damage by ischemia-reperfusion injury, although the greatest effect was observed after 72 hours.

KEY WORDS: Ischemia/reperfusion. Liver transplantation. Ischemic preconditioning. Remote ischemic preconditioning.

\section{Introducción}

El trasplante hepático es considerado como la única opción terapéutica para las enfermedades hepáticas en fase terminal; sin embargo, existen diversos mecanismos que pueden dañar el órgano a trasplantar. El daño por isquemia-reperfusión es un fenómeno de acentuación del daño celular en un órgano isquémico después del restablecimiento del flujo de oxígeno'. En el hígado, el daño por isquemia-reperfusión está asociado al trasplante, la cirugía hepática resectiva o de reconstrucción vascular, y el trauma².

La lesión por isquemia-reperfusión es un proceso complejo y multifactorial en el cual se produce una serie de cambios celulares y moleculares, como activación de la cascada inflamatoria, estrés oxidativo, depleción de energía y un desbalance iónico y de $\mathrm{pH}$ que culmina en el deterioro de la función celular y da lugar a extenso daño tisular ${ }^{3-5}$. La lesión por isquemia-reperfusión es una de las principales causas del retraso en la función inicial y de fallo del injerto hepático.

Numerosas estrategias se han empleado para disminuir el daño por isquemia-reperfusión en diversos modelos experimentales, pero solo algunas de estas han demostrado eficacia en estudios en humanos. En 2003, Selzner, et al. ${ }^{6}$ clasificaron los procedimientos para disminuir la lesión por isquemia-reperfusión en farmacológicos, quirúrgicos (preacondicionamiento isquémico, preacondicionamiento isquémico remoto [PIR]) y terapia génica.

En la actualidad, muchas de las investigaciones en el campo del trasplante van encaminadas a comprender los mecanismos implicados en la lesión por isquemia-reperfusión; la meta es desarrollar nuevas estrategias terapéuticas que permitan disminuir esta lesión y minimizar así el riesgo de disfunción del injerto, pero ninguna de las tres estrategias ha resultado ser totalmente eficaz en la prevención de la lesión por isquemia-reperfusión asociada al trasplante ${ }^{7-9}$.

Dentro de la fisiopatología del PIR se han descrito diversos mecanismos involucrados, entre los cuales está la falla en la bomba de $\mathrm{Na} / \mathrm{K}$, el aumento en la concentración de calcio, la formación de radicales libres, la formación de malondialdehído y la depleción en la capacidad antioxidante ${ }^{10,11}$.

EI PIR es una alternativa terapéutica que se ha utilizado experimentalmente para mejorar la función renal y hepática, y disminuir los cambios inflamatorios tras la reperfusión. El fenómeno de PIR fue descrito en 1993 por Przyklenk, et al..$^{12}$, al observar que episodios breves de isquemia-reperfusión en la arteria circunfleja disminuían el tamaño del infarto del miocardio causado por la oclusión de la arteria descendente anterior izquierda ${ }^{12}$.

Hasta la fecha se han publicado pocos estudios clínicos de PIR, el cual ofrece protección a un órgano o tejido distante ${ }^{13,14}$. El mecanismo por el que un breve episodio de isquemia-reperfusión de un órgano ofrece protección frente a un episodio posterior de isquemia en un órgano o tejido a distancia aún no está claro. Se han propuesto tres teorías para explicar los mecanismos del PIR: la hipótesis neuronal, la hipótesis humoral y una tercera hipótesis que propone que la isquemia transitoria y la reperfusión de un órgano o tejido provocan una respuesta sistémica de protección en el órgano remoto al suprimir la inflamación y la apoptosis mediante la activación de MAPK p38, ERK1/2 y JNK ${ }^{15,16}$. En estudios experimentales se ha investigado el efecto del PIR sobre la respuesta inflamatoria, estimulando la transcripción de genes antiinflamatorios y antiapoptóticos ${ }^{15,17}$. El objetivo de este estudio fue determinar si el PIR modula los mecanismos implicados en la lesión por isquemia-reperfusión en receptores de trasplante hepático de donante cadáver a través de mediadores inflamatorios como las citocinas (IL-1, IL-6, FNT- $\alpha$ ), moléculas de adhesión intracelular (ICAM-1) y factor de crecimiento de epitelio vascular (FCEV).

\section{Método}

\section{Población de pacientes y diseño del estudio}

Se incluyeron 10 pacientes sometidos a trasplante hepático durante el periodo de agosto de 2013 a agosto de 2015 en el Hospital Universitario Dr. José 
Eleuterio González. Los sujetos, de ambos sexos, $>18$ y $<70$ años de edad, sometidos a trasplante hepático no urgente, fueron elegibles para participar en este estudio previo consentimiento informado. El protocolo fue aprobado por el Comité de Ética del Hospital Universitario (TR12-002).

Los 10 receptores fueron divididos en dos grupos: grupo PIR $(n=5)$ y grupo control sin PIR $(n=5)$. EI grupo sanguíneo fue compatible entre los receptores y los donantes. EI PIR fue inducido en el donante por la oclusión del flujo de sangre de ambas extremidades inferiores mediante torniquete neumático colocado simultáneamente en el tercio superior de ambos muslos por 10 minutos $(200 \mathrm{mmHg})$, que se desinfló posteriormente; este procedimiento se realizó inmediatamente antes de comenzar la laparotomía. Tras la procuración del injerto hepático, que se llevó a cabo mediante la técnica clásica, los injertos fueron preservados con solución HTK (Custodiol ${ }^{\circledR}$ ), y en el receptor se realizó el trasplante hepático utilizando la técnica de piggy back. Los injertos hepáticos se irrigaron con solución salina normal y albúmina a través de la vena porta para eliminar la solución de preservación.

En ambos grupos (PIR y control) se tomaron muestras de sangre $(15 \mathrm{ml})$ en las fases pretrasplante, 90 minutos posreperfusión, a las 12, 24, 48 y 72 horas, y a los 7, 15 y 30 días, y en los donantes antes del PIR y al inicio de la laparotomía. En cada fase se obtuvo una muestra de sangre para la determinación de los parámetros hematológicos y bioquímicos, y para los mediadores de respuesta inflamatoria; las muestra de sangre se centrifugaron a $3000 \mathrm{rpm}$ y el suero se alicuotó y almacenó a $-80^{\circ} \mathrm{C}$ hasta su análisis. El régimen de inmunosupresión fue inducido por un bolo de esteroides durante la cirugía antes de la reperfusión y se mantuvo con tacrolimus, micofenolato de mofetilo y esteroides.

Las variables evaluadas en los receptores fueron edad, sexo, etiología de la enfermedad hepática, escala MELD, puntuación de Child-Pugh, tiempo de isquemia fría y tiempo de fase anhepática. El criterio principal de valoración de la eficacia de parte del estudio fue la evaluación de la modificación de los mediadores inflamatorios y de la función hepática a través de la AST, la ALT, la bilirrubina total y la razón normalizada internacional a los 90 minutos de la reperfusión y en las fases posteriores analizadas. Los criterios de valoración secundarios fueron la incidencia de fallo primario del injerto y la mala función inicial del injerto, la necesidad de retrasplante y la supervivencia del injerto a los 3 meses.

\section{Determinación de los parámetros hematológicos y bioquímicos}

Se utilizaron muestras de sangre para determinar los parámetros hematológicos en un Cell Dyn 1700 (Abbott Diagnostics, Abbott Park, IL, EE.UU.) y las concentraciones séricas de los diversos marcadores bioquímicos con kits de ensayos bioquímicos comerciales estándar, utilizando el analizador ILAB Aries (Instrumentation Laboratory Headquarters Bedford, MA, EE.UU.) y DT6011 (Sistema Vitros química, Johnson y Johnson, EE.UU.).

\section{Mediadores proinflamatorios}

Las concentraciones séricas de IL-6 (pg/ml), FNT- $\alpha$ (pg/ml), ICAM-1 (pg/ml), FCEV (pg/ml) e IL- 1 (pg/ml) se determinaron utilizando un kit de ensayo inmunoenzimático específico para cada uno de los mediadores (Peprotech, México).

\section{Análisis estadístico}

Se utilizó el paquete estadístico SPSS 22.0 (SPSS Inc. Software, Chicago, Illinois, EE.UU.) para analizar los datos mediante la prueba t de Student, para determinar la comparación y la diferencia entre los grupos. Todos los valores se expresaron como media \pm desviación estándar, y se consideró estadísticamente significativo un valor de $\mathrm{p}<0.05$.

\section{Resultados}

Los donantes fueron hombres $(4,40 \%)$ y mujeres $(6,60 \%)$, el promedio de edad fue de $41.3 \pm 14.6$ años y en ambos grupos no tenían criterios expandidos; las causas de los fallecimientos fueron traumatismo craneoencefálico (46\%), evento cerebrovascular (45\%) e hipoxia cerebral (9\%).

Los receptores fueron hombres $(6,60 \%)$ y mujeres (4, 40\%), con un promedio de edad de $59.0 \pm 9.7$ años, un índice de masa corporal de $26.1 \pm 5.7$, un valor medio de MELD de 15 y clasificación Child Pugh B (9) y C (1). La etiología de la cirrosis hepática fue alcohólica $(3,30 \%)$, esteatohepatitis no alcohólica $(3,30 \%)$, autoinmunitaria $(1,10 \%)$, hepatitis por virus $C(2,20 \%)$ y cirrosis biliar secundaria $(1,10 \%)$. 


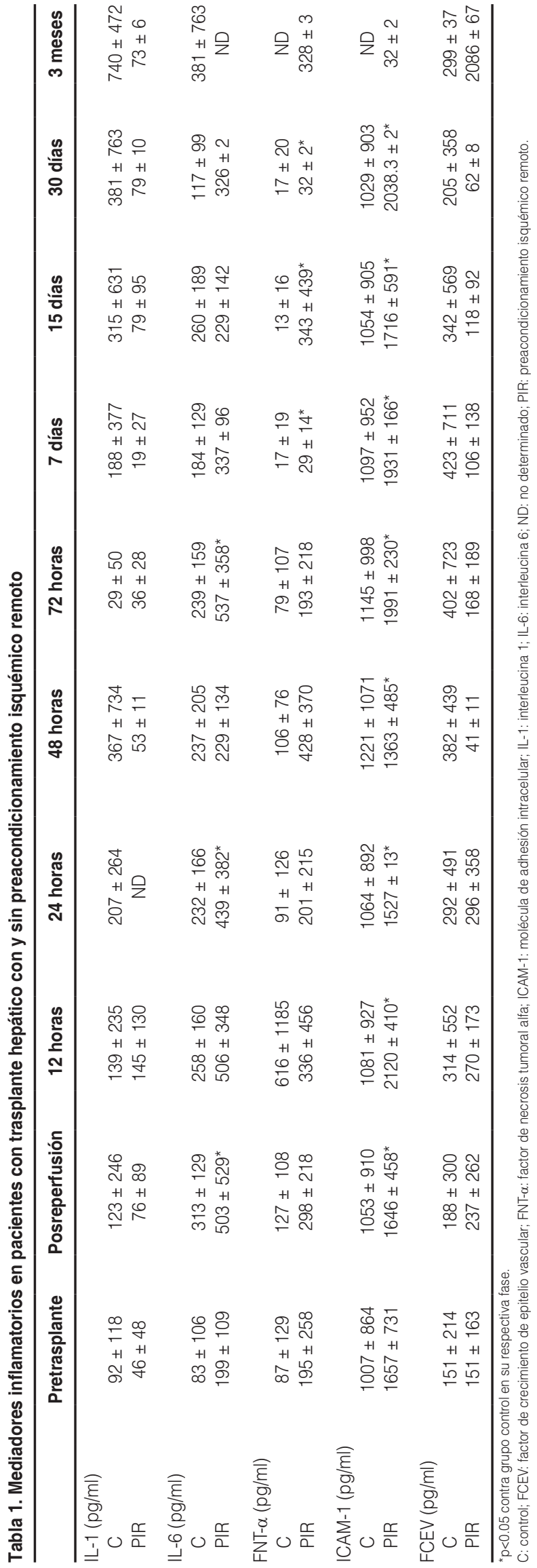

El tiempo promedio de isquemia fría fue de 4.1 horas, y el promedio de la fase anhepática fue de 49 minutos. No hubo ningún caso de falla primaria del injerto, pobre función inicial del injerto ni rechazo agudo. Se encontró diferencia significativa $(p<0.05)$ entre los grupos en los siguientes mediadores inflamatorios y fases: IL-6 en posreperfusión, 24 horas y 72 horas; FNT- $\alpha$ en 7 días, 15 días y 30 días; e ICAM-1 en la mayoría de las fases: Los valores de cada uno de estos mediadores fueron más altos en el grupo que recibió órganos sometidos a PIR; no hubo diferencia significativa en IL-1 y FCEV en todas las fases de estudio (Tabla 1).

Respecto a los parámetros hematológicos evaluados, solo las plaquetas mostraron principalmente diferencia significativa en algunas de las fases evaluadas; sin embrago, se observaron valores más altos en el grupo PIR desde la fase 24 horas (Tabla 2). En los parámetros bioquímicos no se encontró diferencia significativa en la mayoría de las fases (Tabla 3), pero la AST, la ALT y la fosfatasa alcalina fueron más altas en las primeras fases del estudio en el grupo PIR (desde posreperfusión hasta 72 horas) y posteriormente disminuyeron en las siguientes fases, aunque solo fue significativa a los 30 días. La ALT y la AST se normalizaron a los 3 meses en ambos grupos y la fosfatasa alcalina solo en el grupo PIR. Las bilirrubinas disminuyeron significativamente solo en las últimas fases del estudio en el grupo PIR (15 días hasta 3 meses), y se normalizaron en este grupo a partir de los 30 días (Tabla 4).

\section{Discusión}

Se han evaluado diversas estrategias quirúrgicas para disminuir la lesión por isquemia-reperfusión, y entre ellas el preacondicionamiento isquémico ha demostrado que protege frente a la lesión por isquemia-reperfusión ${ }^{18}$; sin embargo, la principal limitante de este procedimiento es la acción directa sobre el órgano blanco y sus estructuras vasculares, por lo que la aplicación clínica del preacondicionamiento isquémico en el trasplante hepático se ha visto limitada por los episodios isquémicos imprevistos y por posibles razones éticas. En cambio, el PIR puede dar protección a órganos distantes sin causarles estrés directo, y además puede aplicarse antes del inicio de la isquemia en el órgano blanco, durante la isquemia final o después de la reperfusión ${ }^{19}$.

Experimentalmente se ha demostrado que el PIR puede reducir la lesión por isquemia-reperfusión 


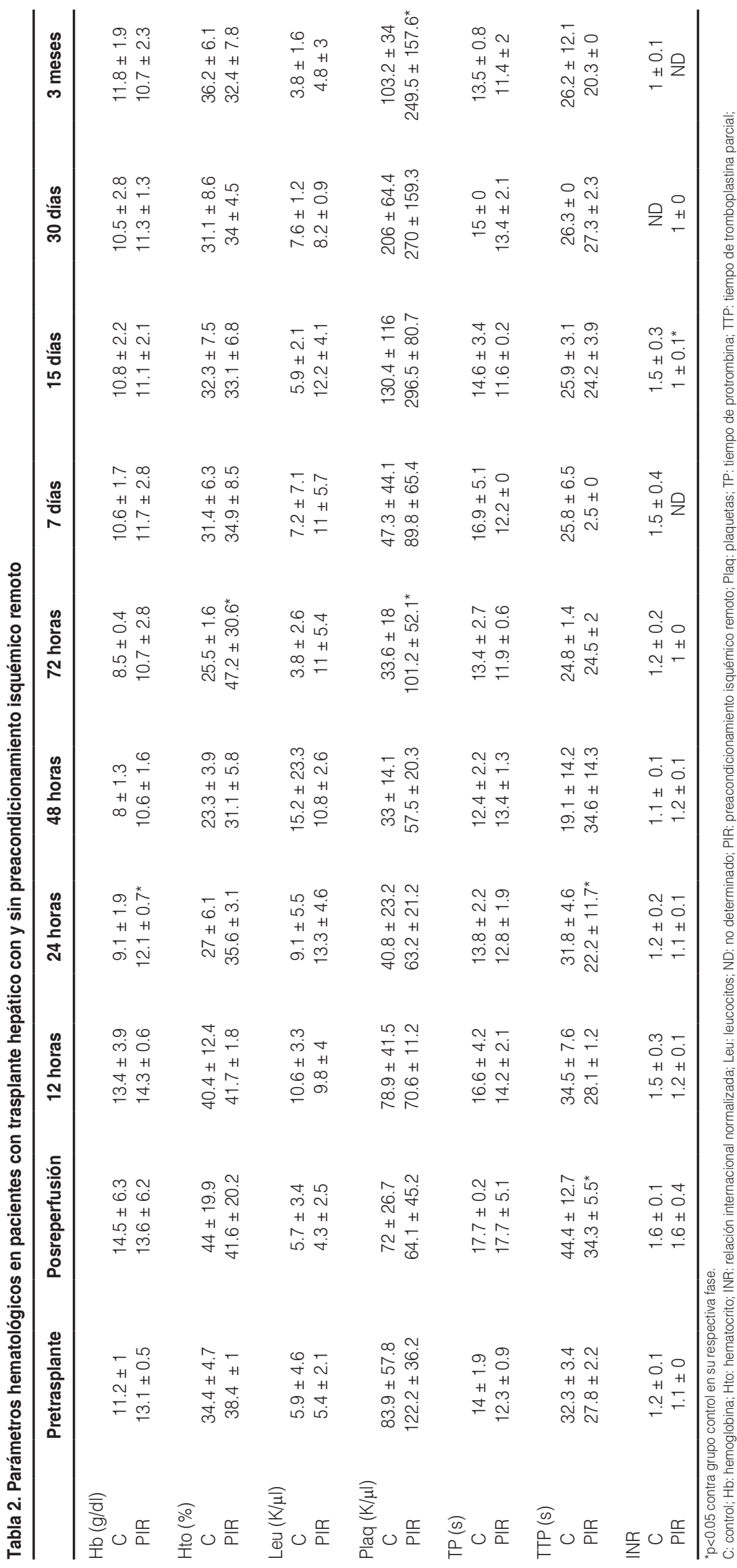

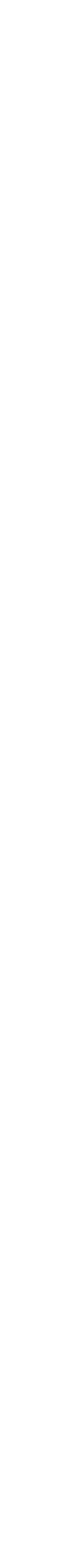




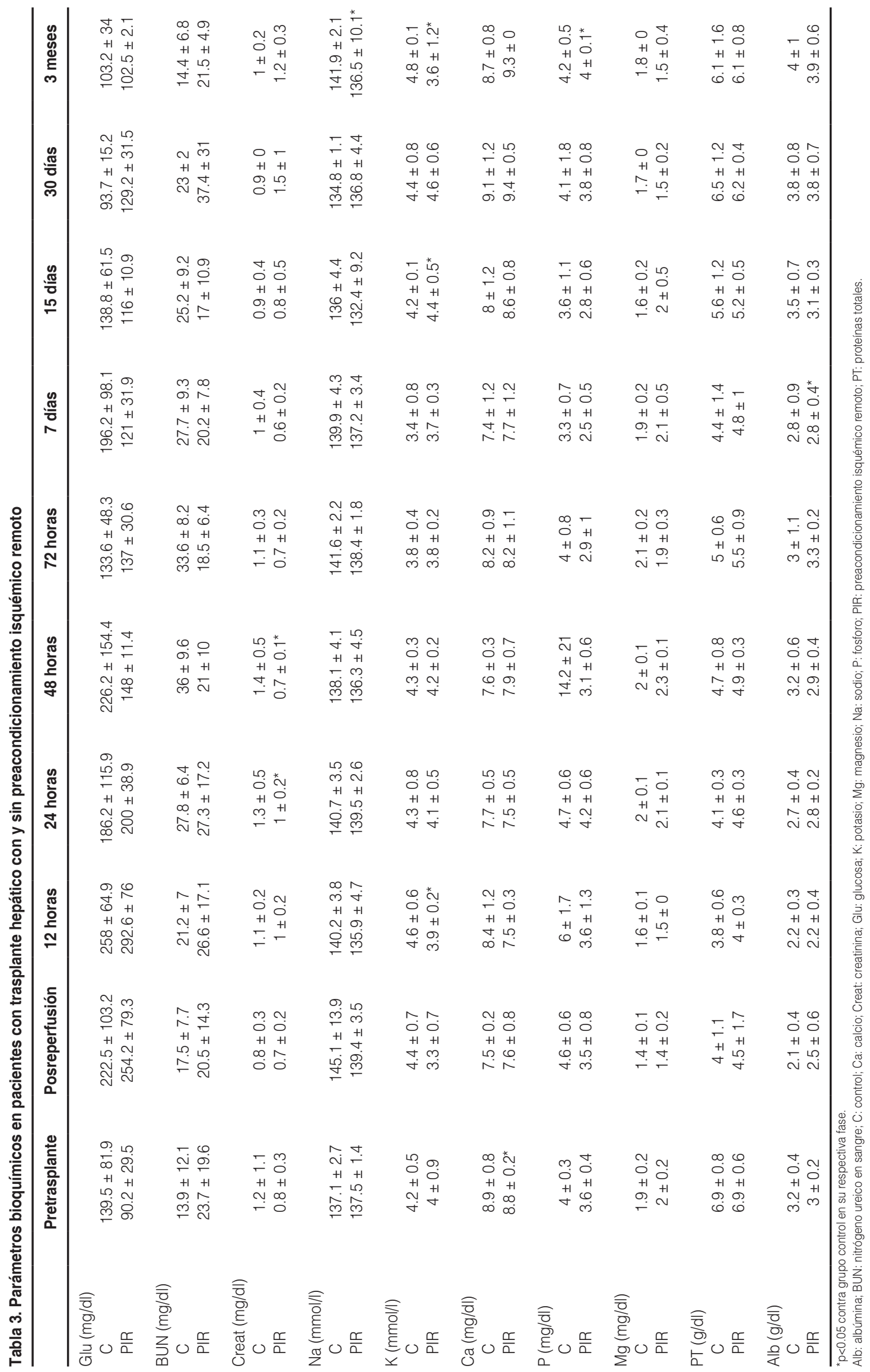




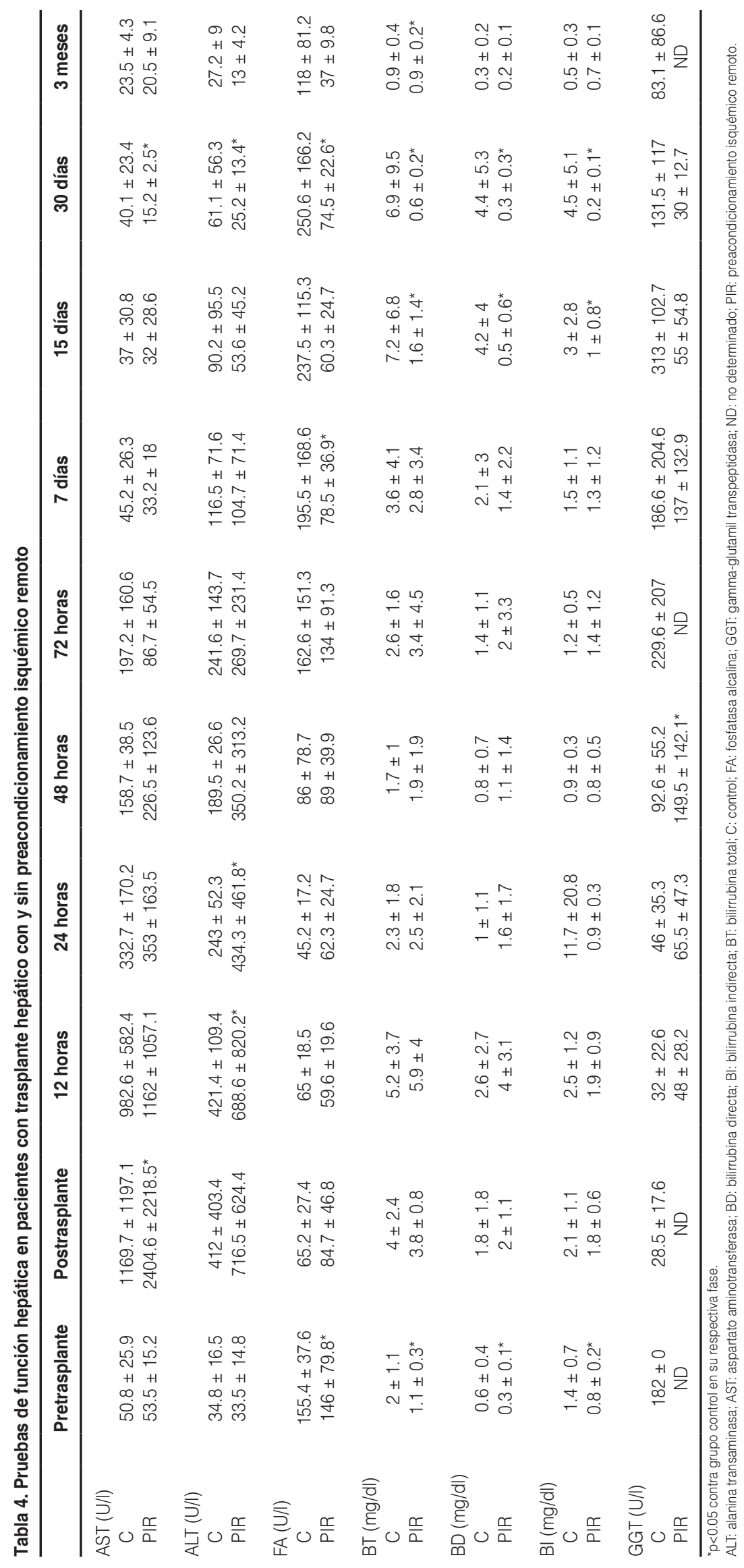


hepática ${ }^{4,20}$; sin embargo, aún existen controversias respecto a la duración de la fase inicial de isquemia, el tiempo de reperfusión y el número de ciclos aplicados $^{21}$. Se ha demostrado, en un modelo de trasplante hepático experimental previo PIR con la aplicación de tres ciclos de isquemia de 5 minutos, que se disminuyen el estrés oxidativo y la inflamación ${ }^{22}$.

No se han publicado estudios clínicos en pacientes con trasplante hepático de cadáver sometidos a PIR; solo se ha reportado un trabajo de trasplante hepático de donante vivo en el que se observó una disminución significativa de la ALT en los primeros 28 días del trasplante en receptores sometidos a PIR posreperfusión, y un aumento significativo en la creatinina sérica y de la tasa de filtración glomerular en el grupo PIR, lo que demostró que el PIR disminuyó la lesión renal aguda; no se observó ningún beneficio clínico relacionado sobre la función temprana del injerto hepático, la frecuencia de complicaciones, la estancia hospitalaria ni la mortalidad ${ }^{23}$.

En la mayoría de los trabajos clínicos en los que se ha empleado la técnica quirúrgica de preacondicionamiento isquémico para disminuir el daño por isquemia-reperfusión en el trasplante hepático se ha reportado una disminución de las aminotransferasas ${ }^{8,9} ; \sin$ embargo, en estudios experimentales el preacondicionamiento isquémico atenúa la lesión por isquemia-reperfusión hepática al disminuir la infiltración de leucocitos, las enzimas hepáticas y la apoptosis ${ }^{24,25}$. En el presente estudio se observó un aumento significativo de dichas enzimas en las fases tempranas del postrasplante hepático, por lo que podemos deducir que en esta etapa el PIR no logró mejorar la lesión por isquemia-reperfusión, pero en las fases subsecuentes evaluadas (después de 72 horas) se observó una mejor respuesta en los sujetos que recibieron un trasplante hepático sometido a PIR.

Recientemente, en un modelo múrido de trasplante hepático con preacondicionamiento isquémico, PIR o ambos, se logró disminuir la lesión por isquemia-reperfusión en los grupos preacondicionados, ya que tuvieron una disminución significativa de las transaminasas, del FNT- $\alpha$ y del malondialdehído, con menor lesión hepática y apoptosis, respecto al grupo control, demostrando un efecto sinérgico en el grupo de preacondicionamiento isquémico más $\mathrm{PIR}^{26}$.

Por otro lado, la trombocitopenia es muy común durante la fase de reperfusión del trasplante hepáti$\mathrm{CO}^{27,28}$, ocasionada por el consumo y el secuestro de las plaquetas dentro de los sinusoides hepáticos. El grado de activación plaquetaria se ha correlacionado con la función del injerto; aunque se cree que las plaquetas participan activamente en la patogénesis de la lesión por isquemia-reperfusión, pueden tener efectos benéficos durante esta fase al secretar sustancias como la serotonina, la cual participa en la reparación del daño isquémico ${ }^{29}$. Existen evidencias que sugieren que las plaquetas tienen otras propiedades no hemostáticas, al participar en la inflamación, la angiogénesis, la regeneración y la lesión por isquemia-reperfusión $^{30}$. Se ha demostrado experimentalmente que el PIR protege contra la isquemia hepática parcial a través de la serotonina derivada de las plaquetas, el FCEV, la IL-10 y la matriz de metaloproteinasa $8^{30}$. En este estudio se encontró una mayor recuperación del número de plaquetas a partir de las 72 horas, la cual persistió hasta 3 meses en el grupo de pacientes receptores de injertos sometidos a PIR.

Hasta la fecha solo se ha reportado el efecto del PIR en receptores de trasplante hepático de donante vivo relacionado. Este es el primer estudio en el cual se procede a la comparación de injertos hepáticos sometidos a PIR con injertos no sometidos a este en donantes con muerte encefálica. En este trabajo se observó que, de los mediadores inflamatorios evaluados, solo la IL-6 parece participar en las fases tempranas de la respuesta inflamatoria postrasplante hepático, contrario al FNT- $\alpha$, cuya participación se evidencia después del séptimo día postrasplante; sin embargo, la ICAM-1 parece ser un mediador inflamatorio más notorio en los receptores que recibieron injertos con PIR, ya que esta molécula se elevó significativamente desde la fase inicial postrasplante hepático hasta los 30 días, y normalizándose solo hasta los 3 meses.

Es de notar que, a la par de los mediadores inflamatorios, las enzimas hepáticas AST, ALT y fosfatasa alcalina se elevan considerablemente en las fases postrasplante inmediato y disminuyen más rápido en los receptores con PIR a partir de las 72 horas, manteniéndose dicho efecto hasta los 15 días y logrando recuperar valores normales con más rapidez en dichos sujetos, y no así en el grupo de receptores sin PIR. En ambos grupos de estudio no se presentó falla primaria ni rechazo del injerto durante el seguimiento evaluado, pero sí se observó que en el grupo sometido a PIR se logró una recuperación más rápida tanto de los parámetros inflamatorios como de algunos parámetros bioquímicos asociados a una mejoría de la función hepática, por lo que consideramos que el procedimiento favorece la disminución del daño por isquemia-reperfusión en los injertos hepáticos, y que 
es necesario incrementar el número de pacientes y definir a través de qué mecanismos se atribuye dicha mejoría.

\section{Financiamiento}

CONACYT-México (2012-1-182653) y PAICYT-UANL.

\section{Conflicto de intereses}

Los autores declaran que no existen conflictos de intereses.

\section{Bibliografía}

1. Teoh N, De la Pena A, Farrell G. Hepatic ischemic preconditioning in mice is associated with activation of NFuB, p38 kinase, and cell cycle entry. Hepatology. 2002;36:94-102.

2. Ramírez P, Marín JM, Piñero A, Chávez-Cartaya R, Parrilla P. Investigación experimental aplicada a la clínica: isquemia-reperfusión hepática. Cir Esp. 2000;67:281-91.

3. Jaeschke H. Molecular mechanisms of hepatic ischemia-reperfusion injury and preconditioning. Am J Physiol Gastrointest Liver Physiol. 2003;284:15-26.

4. Wang F, Birch SE, He R, Tawadros P, Szaszi K, Kapus A, et al. Remote ischemic preconditioning by hindlimb occlusion prevents liver ischemic/reperfusion injury: the role of High Mobility Group-Box 1. Ann Surg. 2010;251:292-9.

5. Selzner M, Selzner N, Jochum W, Graf R, Clavien PA. Increased ischemic injury in old mouse liver: an ATPudependent mechanism. Liver Transpl. 2007;13:382-90.

6. Selzner N, Rudiger H, Graf R, Clavien PA. Protective strategies against ischemic injury of the liver. Gastroenterology. 2003;125:917-36.

7. Peralta C, Serafín A, Fernández-Zabalegui L, Wu ZY, Roselló-Catafau J. Liver ischemic preconditioning: a new strategy for the prevention of ischemia-reperfusion injury. Transplant Proc. 2003;35:1800-2.

8. Azoulay D, Del Gaudio M, Andreani P, Ichai P, Sebag M, Adam R, et al. Effects of 10 minutes of ischemic preconditioning of the cadaveric liver on the graft's preservation and function: the ying and the yang. Ann Surg. 2005;242:133-9.

9. Cescon M, Grazi GL, Grassi A, Ravaioli M, Vetrone G, Ercolani G, et al. Effect of ischemic preconditioning in whole liver transplantation from deceased donors. A pilot study. Liver Transpl. 2006;12:628-35.

10. Costa FL, Yamaki VN, Gonçalves TB, Coelho JV, Percário S, Brito MV. Combined remote ischemic perconditioning and local postconditioning on liver ischemia-reperfusion injury. J Surg Res. 2014;192:98-102.

11. Eltzschig HK, Eckle T. Ischemia and reperfusion — from mechanism to translation. Nat Med. 2011;17:1391-401.
12. Przyklenk K, Bauer B, Ovize M, Kloner RA, Whittaker P. Regional ischemic' preconditioning protects remote virgin myocardium from subsequent sustained coronary occlusion. Circulation. 1993;87:893-9.

13. Wu J, Feng $X$, Huang $H$, Shou $Z$, Zhang $X$, Wang $R$, et al. Remote ischemic conditioning enhanced the early recovery of renal function in recipients after kidney transplantation: a randomized controlled trial. J Surg Res. 2014;188:303-8.

14. Nicholson ML, Pattenden CJ, Barlow AD, Hunter JP, Lee G, Hosgood SA. A double blind randomized clinical trial of remote ischemic conditioning in live donor renal transplantation. Medicine. 2015;94:e1316.

15. Le Page S, Prunier F. Remote ischemic conditioning: current clinical perspectives. J Cardiol. 2015;66:91-6.

16. Hausenloy DJ, Yellon DM. Remote ischemic preconditioning: underlying mechanisms and clinical application. Cardiovasc Res. 2008;79:377-86.

17. Konstantinov IE, Arab S, Li J, Coles JG, Boscarino C, Mori A, et al. The remote ischemic preconditioning stimulus modifies gene expression in mouse myocardium. J Thorac Cardiovasc Surg. 2005;130:1326-32.

18. Koneru B, Fisher A, He Y, Klein KM, Skurnick J, Wilson DJ, et al. Ischemic preconditioning in deceased donor liver transplantation: a prospective randomized clinical trial of safety and efficacy. Liver Transpl. 2005; 11:196-202

19. Jia JJ, Li JH, Jiang L, Lin BY, Wang L, Su R, et al. Liver protection strategies in liver transplantation. Hepatobiliary Pancreat Dis Int. 2015; 14:34-42.

20. Czigany Z, Turoczi Z, Bulhardt O, Hegedus V, Lotz G, Rakonczay Z, et al. Remote ischemic conditioning: short-term effects on rat liver ischemic-reperfusion injury. Orv Hetil. 2012;153:1579-87.

21. Skyschally A, van Caster P, Iliodromitis EK, Schulz R, Kremastinos DT, Heusch G. Ischemic postconditioning: experimental models and protocol algorithms. Basic Res Cardiol. 2009;104:469-83.

22. Jia J, Li J, Jiang L, Zhang J, Chen S, Wang L, et al. Protective effect of remote limb ischemic perconditioning on the liver grafts of rats with a novel model. PLoS One. 2015;10:e0121972.

23. Kim WH, Lee JH, Ko JS, Min JJ, Gwak MS, Kim GS, et al. Effect of remote ischemic postconditioning on patients undergoing living donor liver transplantation. Liver Transpl. 2014;20:1383-92.

24. Jin LM, Liu YX, Zhou L, Xie HY, Feng XW, Li H, et al. Ischemic preconditioning attenuates morphological and biochemical changes in hepatic ischemia/reperfusion in rats. Pathobiology. 2010;77:136-46.

25. Yan S, Jin LM, Liu YX, Zhou L, Xie HY, Zheng SS. Outcomes and mechanisms of ischemic preconditioning in liver transplantation. Hepatobiliary Pancreat Dis Int. 2010;9:346-54.

26. Li DY, Shi XJ, Li W, Sun XD, Wang GY. Ischemic preconditioning and remote ischemic preconditioning provide combined protective effect against ischemia/reperfusion injury. Life Sci. 2016;1:76-80.

27. Novaković-Anucin S, Gnip S, Canak V, Jurisić D, Radović P, Erdeljan S, et al. Laboratory monitoring of the haemostatic system changes during orthotopic liver transplantation. Srp Arh Celok Lek. 2013;141:608-14.

28. Hartmann M, Szalai C, Saner FH. Hemostasis in liver transplantation: pathophysiology monitoring and treatment. World J Gastroenterol. 2016;22:1541-50.

29. Pereboom IT, Lisman T, Porte RJ. Platelets in liver transplantation: friend or foe? Liver Transpl. 2008;14:923-31.

30. Tripodi A, Salerno F, Chantarangkul V, Clerici M, Cazzaniga M, Primignani $\mathrm{M}$, et al. Evidence of normal thrombin generation in cirrhosis despite abnormal conventional coagulation tests. Hepatology. 2005;41:553-8. 